\title{
Secondary electron emission in a photocathode rf gun
}

\author{
J.H. Han* and M. Krasilnikov \\ DESY, Platanenallee 6, 15738 Zeuthen, Germany
}

\section{K. Flöttmann}

DESY, Notkestrasse 85, 22603 Hamburg, Germany

(Received 3 December 2004; published 8 March 2005)

\begin{abstract}
During the last decades, photocathode rf guns have been proven to be successful for providing very high quality electron beams required for vacuum ultraviolet and $\mathrm{x}$-ray free-electron lasers. Beam dynamics simulations show that the electron beam quality in a rf gun depends strongly on the beam dynamics in the vicinity of the cathode. Therefore, the injection process plays a significant role in the beam performance. Several codes are available to simulate the beam dynamics in the gun. They are able to track the beam under the influence of external fields and space charge forces, but details of the emission processes are still missing in these simulations. In photocathode rf guns, the electron beams have a high charge density. Especially during emission from the cathode, the electrons have a very low velocity and experience high longitudinal space charge forces counteracting the applied accelerating field. Because of the space charge field, some part of the electrons emitted from the cathode might move backward to the cathode where they can produce secondary electrons. A high electric field in the gun cavity, on the other hand, generates a large amount of dark current. If the field-emitted electrons from the cathode or any other surface inside the cavity hit the cathode, secondary electrons can be produced as well. For a detailed understanding of the electron beam and dark current in a rf gun, simulations including a model of the secondary electron emission are necessary. In this paper, a simple model is discussed with an application to the beam dynamics at high emission phases in rf guns. Detailed simulations have been done in comparison to measurements at the Photo Injector Test Facility at DESY in Zeuthen. The primary electrons which are photoemitted from the cathode and the secondary electrons which are produced by the primaries at the cathode could be clearly distinguished in measurements and simulations.
\end{abstract}

DOI: 10.1103/PhysRevSTAB.8.033501

PACS numbers: 29.25.Bx, 41.75.Fr, 79.20.Hx

\section{INTRODUCTION}

Photocathode rf guns have been proven to be an optimum electron source for very high quality beams required for vacuum ultraviolet and x-ray free-electron lasers (FELs) [1,2]. In a photocathode rf gun, the electron beam is generated by a laser pulse with a well-defined transverse and temporal profile. This electron beam is immediately accelerated to become relativistic by a high rf field, thereby keeping the transverse emittance small. The electron beam dynamics after extraction from the cathode is well understood analytically $[3,4]$ and numerically. Several codes, for instance ASTRA [5] and PARMELA [6], have been successfully used to simulate the beam dynamics in rf guns.

Nevertheless, the emission mechanism from the photocathode rf gun is not fully understood yet. For example, a long-term variation of the photoemission and the field emission properties from a $\mathrm{Cs}_{2} \mathrm{Te}$ photocathode has been found at the Fermilab A0 photoinjector [7]. This behavior is closely related to the solenoid field configuration at the cathode but not to the driving laser. For the so-called round beam configuration, the magnetic solenoid field is compensated to be zero at the cathode, while for the so-called flat beam configuration a high magnetic solenoid field is

*Electronic address: jang.hui.han@desy.de applied at the cathode [8]. Switching from the round beam configuration to the flat beam configuration, both the quantum efficiency and the dark current decrease within roughly $1 \mathrm{~h}$, while both recover to the original values when switching back to the round beam configuration. The main reason of this slow dependence is thought to be different bombardment characteristics of the field-emitted electrons onto the $\mathrm{Cs}_{2} \mathrm{Te}$ cathode, which changes the surface properties of the cathode. Hartung et al. [7] found also that multipacting in the $\mathrm{A} 0$ gun cavity shows a relation to the solenoid configuration and the material of the photocathode. Such a behavior was not found with a molybdenum cathode, which has a secondary emission yield less than one except for a narrow energy range $(300 \mathrm{eV}-2 \mathrm{keV})$ of primary electrons [9].

As a step toward a more precise numerical simulation of the emission mechanisms in photocathode rf guns, an algorithm for the secondary electron emission has been modeled and implemented into ASTRA. The employed secondary emission model is briefly discussed in Sec. II. If the emission phase of the electrons is varied, the electron dynamics becomes unstable and even chaotic in some phase intervals, because the electron velocity stays low and the electrons slip with respect to the rf phase. As an application of the secondary emission model, this unstable phase range is studied in detail. After the discussion of the 
single electron dynamics, the measured electron beam characteristics are analyzed by means of a numerical simulation with ASTRA. In Sec. III, the primary and secondary electron dynamics is discussed. In the following section, the dependence of the secondary emission properties on the cathode parameters is studied by comparison of two cathodes.

\section{SECONDARY ELECTRON EMISSION}

When a primary electron strikes a solid material, it may penetrate the surface and generate secondary electrons. The origin of secondary electrons is separated into the following three categories [10]: When the primary electron is reflected off the surface, it is called "backscattered secondary." If the electron penetrates the surface and scatters off one or more atoms and is reflected back out, it is a "rediffused secondary." If the electron interacts inelastically with the material and releases more electrons, "true secondary electrons" are generated. These secondary generation processes are sketched in Fig. 1.

When the primary electrons have an energy of more than several $\mathrm{keV}$, the true secondary electrons are dominant [10]. In the rf gun cavity, the primary electrons typically have a high energy over several hundred $\mathrm{keV}$ because of a rf field of the order of $10 \mathrm{MV} / \mathrm{m}$. Therefore only true secondary electrons are considered here.

True secondary electron emission occurs in the following three steps [11]: (1) production of internal electrons by bombardment of the primary electrons; (2) transport of the internal secondary electrons toward the surface, which is accompanied by some energy loss due to inelastic scattering; and (3) escape of the electrons through the solidvacuum interface.

The average number of internal electrons produced by the primary electrons (step 1) is proportional to the impact energy of the primary electrons divided by the energy required to make an electron-hole pair [12]. The probability of the transport toward the surface (step 2) is

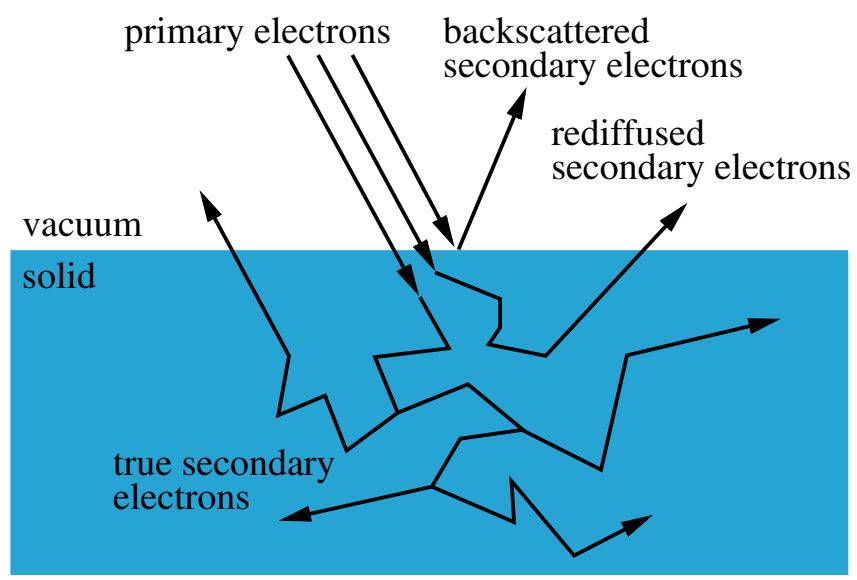

FIG. 1. (Color) Secondary electron generation processes. related to the penetration depth of the primary electron and the mean free path of the secondary electrons, while the probability that an electron which reaches the surface escapes from the solid (step 3) is a function of the energy of the electron divided by the electron affinity of the emissive material [12].

The secondary electron emission process shows great similarities to the photoemission process, which is discussed in an equivalent three step model. Differences arise due to the primary process [penetration depth of $(\sim \mathrm{eV})$ photons compared to the penetration depth of $(\sim \mathrm{keV})$ electrons] and differences of the secondary electron and photoelectron energies, respectively. In $\mathrm{Cs}_{2} \mathrm{Te}$, photoelectrons are produced into the so-called magic window, i.e., their energy is too low to produce electron-hole pairs by electron-electron scattering and hence they do not lose energy during the transport to the surface. Secondary electrons, on the other hand, start with higher energy but lose effectively their energy on the way to the surface until they reach the magic window. Despite these differences, good photoemitters are in general good secondary emitters [13] and the surface properties which affect the photoemission can also affect the secondary emission.

The complicated secondary electron emission process is summarized in an empirical fit [10] which relates the energy of the primary electrons to the number of secondary electrons escaping from the surface:

$$
\delta\left(E_{p}\right)=\delta_{\max } \frac{E_{p}}{E_{p, \max }} \frac{s}{s-1+\left(E_{p} / E_{p, \max }\right)^{s}},
$$

where $\delta\left(E_{p}\right)$ is the secondary electron emission yield depending on the energy of the primary electron, $\delta_{\max }$ is the maximum secondary yield, which occurs at the primary electron energy $E_{p \text {,max }}$, and $s$ is a fit parameter, larger than 1 , which describes the form of the secondary emission yield curve. Figure 2 shows the relative secondary yield for various $s$.

When an electron hits a geometrical aperture in ASTRA, the program generates a random integer number of secondaries according to this model function using a Poisson generator. The energy of the secondaries is assumed to have a Gaussian distribution with a few $\mathrm{eV}$ width $[14,15]$. However, the initial energy as well as the emission angle of the secondaries does not affect the beam dynamics in the gun cavity because the energy is relatively small compared to the energy gain by the accelerating field at the cathode.

The time delay of the secondary emission is assumed to be negligibly small in comparison with the rf cycle of the cavity [16]. The incidence angle effect of the secondary emission yield is not considered because electron bombardment at the cavity is almost normal direction to the surface especially for the backplane of the cavity.

To the authors' knowledge, no measured data for the secondary electron emission properties of $\mathrm{Cs}_{2} \mathrm{Te}$ are pub- 


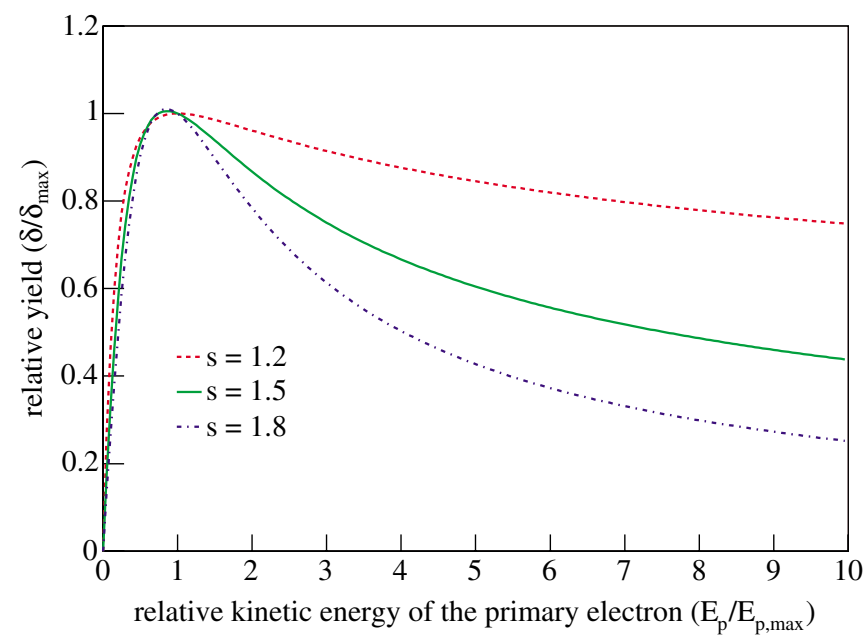

FIG. 2. (Color) Secondary electron emission yield curves according to Eq. (1).

lished yet. But the parameters can be estimated from the data of similar materials such as CsI. CsI has a maximum secondary yield of 17.23 at a primary electron energy of $2.15 \mathrm{keV}[17,18]$. This high secondary yield is explained by the high electronic band gap of $6.3 \mathrm{eV}$ and low electron affinity of $0.1 \mathrm{eV}$ [17].

$\mathrm{Cs}_{2} \mathrm{Te}$ has also a large band gap of $3.3 \mathrm{eV}$ and a small electron affinity of $0.2 \mathrm{eV}$ [19]. Therefore, $\mathrm{Cs}_{2} \mathrm{Te}$ is expected to have similar secondary emission properties as CsI. Actually, the secondary emission yield is strongly dependent on the surface status of the emissive material. During the gun operation, a quantum efficiency change of the $\mathrm{Cs}_{2} \mathrm{Te}$ photocathodes from $\sim 7 \%$ to $\sim 0.5 \%$ was observed. Measurements presented here are done at the lower quantum efficiency. For the measurements in the following section cathode \#500 [20] has been used. The maximum yield and the corresponding primary electron energy are assumed to be 7.0 and $2.2 \mathrm{keV}$, respectively. The fit parameter $s$ is set to 1.5 . With these parameters the measurement results obtained with cathode \#500 can be nicely matched, as will be shown below. The dependence of the secondary emission yield on cathode parameters is discussed in Sec. IV.

\section{BEAM DYNAMICS INCLUDING SECONDARY EMISSION}

\section{A. Experimental setup}

The experiments presented here have been performed at the Photo Injector Test Facility at DESY in Zeuthen (PITZ) [21]. The PITZ gun and downstream beam line with diagnostic components are schematically shown in Fig. 3. The setup of the experiment consists of a 1.5 cell gun cavity operated at a resonance frequency of $1.3 \mathrm{GHz}$, a coaxial $\mathrm{rf}$ coupler, two solenoids for space charge emittance compensation, a photocathode with a cathode plug $(8 \mathrm{~mm}$ radius) made from molybdenum, partially covered with a photoemissive $\mathrm{Cs}_{2}$ Te film ( $2.5 \mathrm{~mm}$ radius), a $262 \mathrm{~nm}$ driving laser with an adjustable transversal and temporal profile, and various diagnostics components for beam charge, size, momentum, and emittance measurements. PITZ aims to produce high density electron beams with small transverse emittance and short bunch length as required for FEL operation.

In order to distinguish the secondary electrons from the primary, i.e., the UV-laser induced photoelectrons, experiments have been performed with the following conditions: A short (2.3 ps rms) Gaussian laser pulse was used in order to observe clearly the rf phase dependence of the electron beam dynamics; a very low charge $(\sim 5 \mathrm{pC})$ was generated with the laser system to get rid of the space charge effects, and a relatively low rf field $(21.6 \mathrm{MV} / \mathrm{m}$ at the cathode) was applied in order to reduce the impact energy of the photoemitted primary electrons and to generate a relatively large number of secondary electrons. The main and bucking solenoids were switched off during charge measurement to simplify the electron beam dynamics and a cathode with a thick $\mathrm{Cs}_{2} \mathrm{Te}$ film (60 nm, cathode \#500) was inserted to maximize the secondary electron production. These parameters are summarized and compared to the normal operating conditions of PITZ in Table I.

\section{B. Single electron dynamics}

The longitudinal electric field distribution inside the rf gun cavity is shown in Fig. 4 with the geometrical aperture of the gun cavity. The gun cavity and the rf coupler are

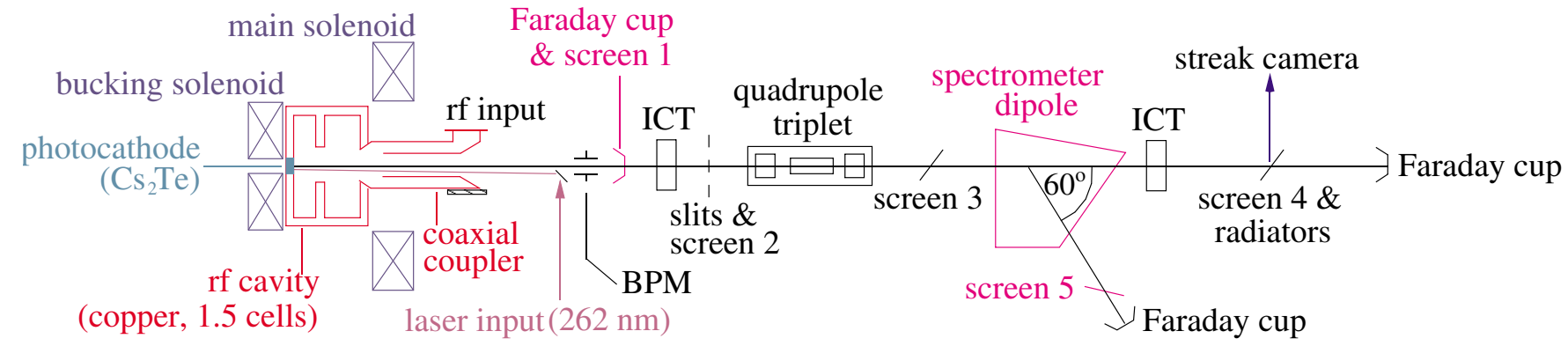

FIG. 3. (Color) Schematic view of the gun and diagnostic sections. 
TABLE I. Machine parameters.

\begin{tabular}{lcc}
\hline \hline & This study & Normal operation \\
\hline Laser profile & $2.3 \mathrm{ps} \mathrm{rms} \mathrm{Gaussian}$ & $\sim 20 \mathrm{ps}$ flattop \\
Bunch charge & $\sim 5 \mathrm{pC}$ & $1 \mathrm{nC}$ \\
Maximum rf field & $21.6 \mathrm{MV} / \mathrm{m}$ & $\gtrsim 40 \mathrm{MV} / \mathrm{m}$ \\
Main solenoid field & $0 \mathrm{~T}$ & $\sim 0.17 \mathrm{~T}$ \\
$\mathrm{Cs}_{2}$ Te thickness & $60 \mathrm{~nm}$ & $30 \mathrm{~nm}$ \\
\hline \hline
\end{tabular}

cylindrically symmetric with respect to the $z$ axis. At the cathode, the longitudinal $\mathrm{rf}$ field oscillates as $E(\phi)=$ $E_{0} \sin \phi$, where $E_{0}$ is the maximum field and $\phi$ is the rf phase. When $\phi$ has a value between $0^{\circ}$ and $180^{\circ}$, the electrons inside the photocathode are extracted out of the cathode by the rf field.

Figure 5 shows the momentum gain of a photoemitted electron in the 1.5 cell $\mathrm{rf}$ cavity as a function of the relative phase of the gun rf field to the laser pulse, i.e., the emission phase. The corresponding accelerating gradient at the cathode is shown together with the simulation. Since the electrons start with zero velocity at the cathode, a strong phase slippage occurs - due to the high gradient mainly within the first centimeters of the half cell — which is responsible for the functional dependency shown in Fig. 5. Figure 6 shows the phase, $\omega t-k z+\phi_{0}$ [3], at three locations, i.e., the entrance, the center, and the exit of the full cell as a function of the emission phase $\phi_{0}$. Electrons starting at $0^{\circ}$ reach already a phase of $65^{\circ}$ at the center of the full cell. They are hence efficiently accelerated in the half and the full cell. Acceleration at the phase of $90^{\circ}$ at the center of the full cell occurs at a starting phase of $48^{\circ}$. Particles starting at $\sim 95^{\circ}$ slip further to a phase of inefficient acceleration in the half cell and end up at $180^{\circ}$ in the full cell. At a phase larger than $\sim 95^{\circ}$, particles are decelerated in the full cell and are eventually stopped. Electrons starting between $\sim 95^{\circ}$ and $\sim 113^{\circ}$ can move forward and

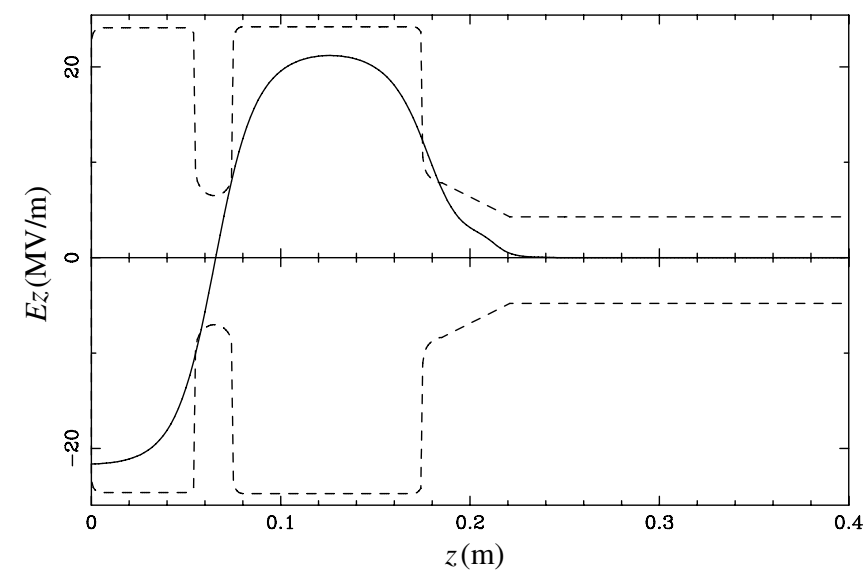

FIG. 4. Longitudinal electric field (solid line) and cavity aperture (dashed line, transversally in arbitrary units) used in the simulations.

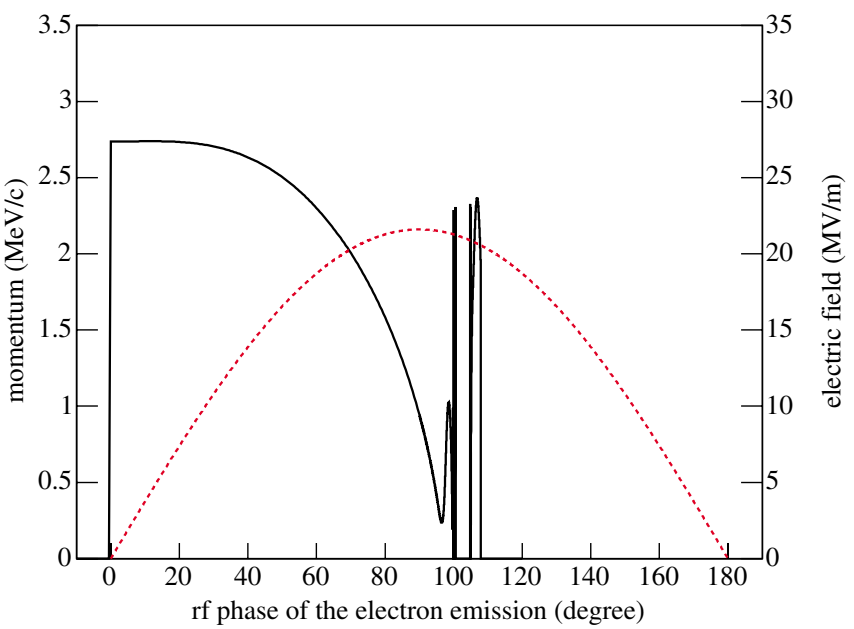

FIG. 5. (Color) Longitudinal electric field on the cathode (red, dotted line) and momentum gain for a single electron (black, solid line) versus rf phase.

backward in the gun, while the phase may slip over several rf cycles. These electrons may hit the cathode, or an aperture, or flow out of the cavity at specific phases.

If the electron travels back and hits the $\mathrm{Cs}_{2} \mathrm{Te}$ photocathode it can generate secondary electrons. If the electron, however, hits the copper cavity or the molybdenum cathode plug it disappears because $\mathrm{Cu}$ and Mo have secondary emission yields below one except for a primary impact energy between several hundred $\mathrm{eV}$ and a few $\mathrm{keV}$. In this study, secondary emission from the cavity surface except the $\mathrm{Cs}_{2} \mathrm{Te}$ photocathode is ignored because such a low impact energy hardly occurs in a cavity with high rf field.

The simulation result of the secondary emission yield in dependence on the emission phase of the primary electron is shown in Fig. 7. For this simulation 10000 identical primary electrons are tracked to generate secondary electrons, so that statistical fluctuations due to the random

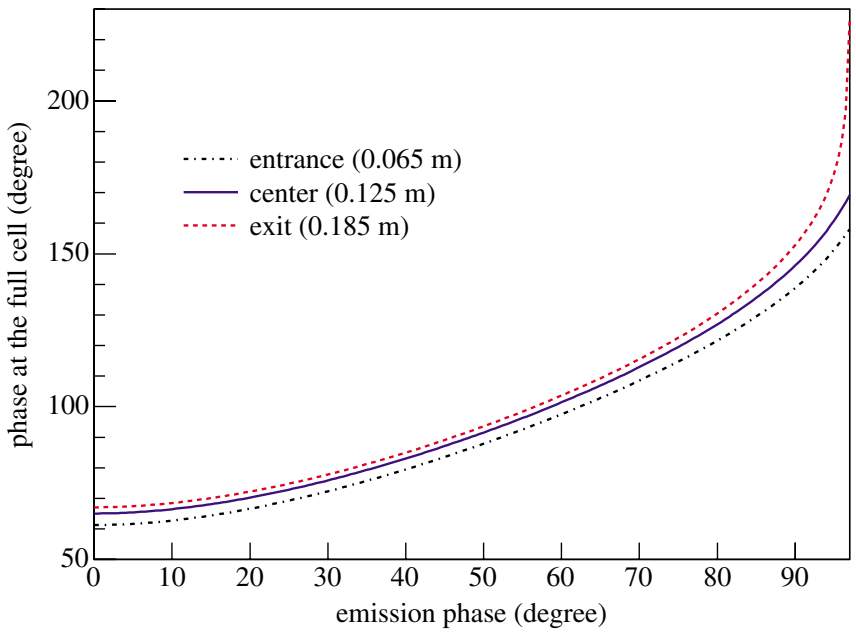

FIG. 6. (Color) Phase at the entrance, center, and exit of the full cell versus emission phase in the gun cavity. 


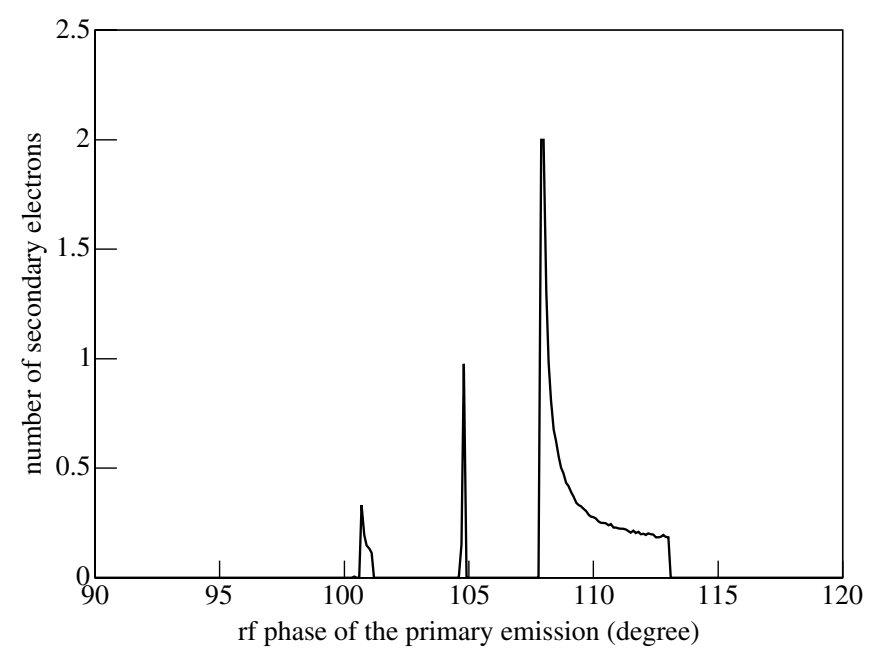

FIG. 7. Average number of secondary electrons generated by a single primary electron as a function of the primary emission phase.

integer generation of the secondaries are averaged out. While the number of generated secondary electrons decreases in the phase range $108^{\circ}-113^{\circ}$, the momentum of the secondaries increases as shown in Fig. 8.

The two parameters responsible for this dependence are the rf phase at which the primary electron hits the cathode (impact phase $=$ launch phase of the secondaries) and the primary electron energy.

Figure 9 shows both parameters for the emission phase range $108^{\circ}-113^{\circ}$ of the primary electron. Around $108^{\circ}$, the impact phase of the photoemitted electrons is $\sim 60^{\circ}$ so that the generated secondary electrons gain a low momen$\operatorname{tum}(\sim 2.3 \mathrm{MeV} / c)$. On the other hand, the impact energy

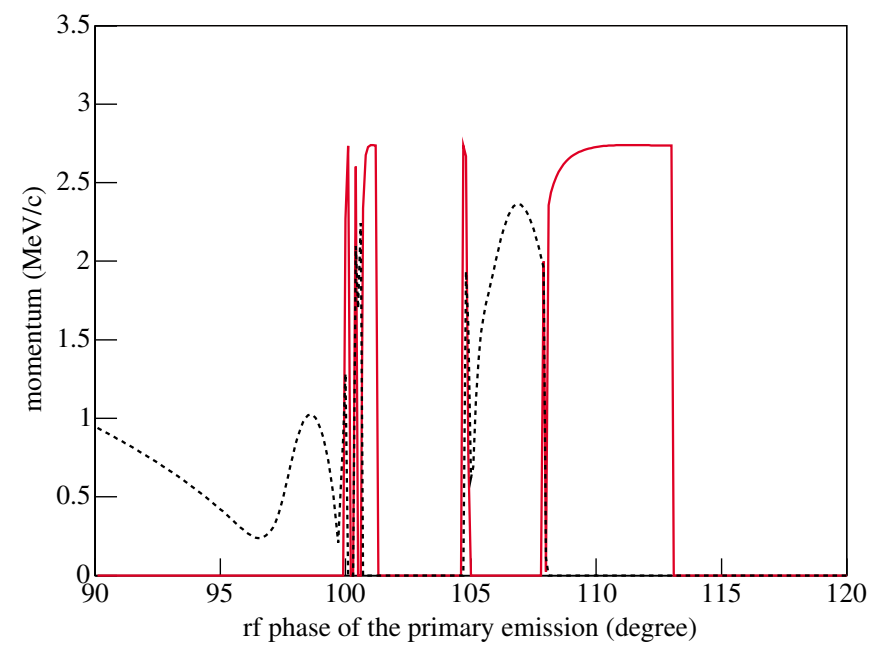

FIG. 8. (Color) Electron dynamics simulation for photoemitted electrons (black, dotted line). The momentum of the electron has been calculated at the exit of the gun. When secondary electrons are produced, the mean momentum of the secondary electrons is plotted (red, solid line).

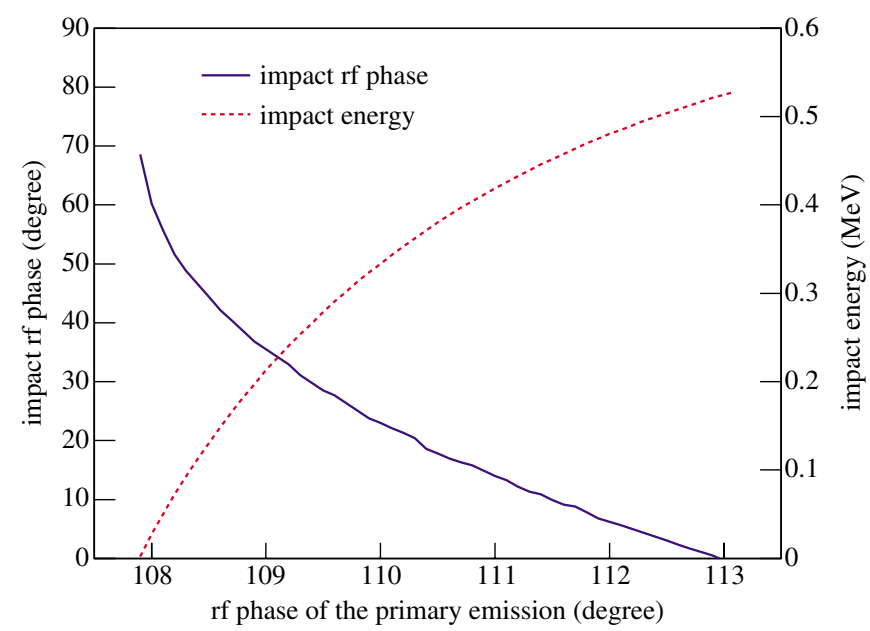

FIG. 9. (Color) A simulation of the primary electron energy and the impact rf phase as a function of the primary emission phase. The generated secondary electrons are delayed, compared to the primary electrons, by one rf cycle.

is very small $(\sim 28 \mathrm{keV})$ so that the secondary electron yield is about two. At a rf phase just below $113^{\circ}$, the secondary electrons gain the maximum momentum because they start at a rf phase close to $0^{\circ}$. But the impact energy is too high to make a large number of secondary electrons. Above $113^{\circ}$ secondary electrons cannot be extracted from the cathode due to the negative impact phases.

\section{Electron beam dynamics}

Figure 10 shows measurements of the beam charge and the mean momentum as a function of the emission phase in comparison to an ASTRA simulation. For the charge measurement, a Faraday cup located $0.78 \mathrm{~m}$ downstream of the cathode has been used. For the momentum measurements, a spectrometer dipole $3.45 \mathrm{~m}$ downstream and a yttrium

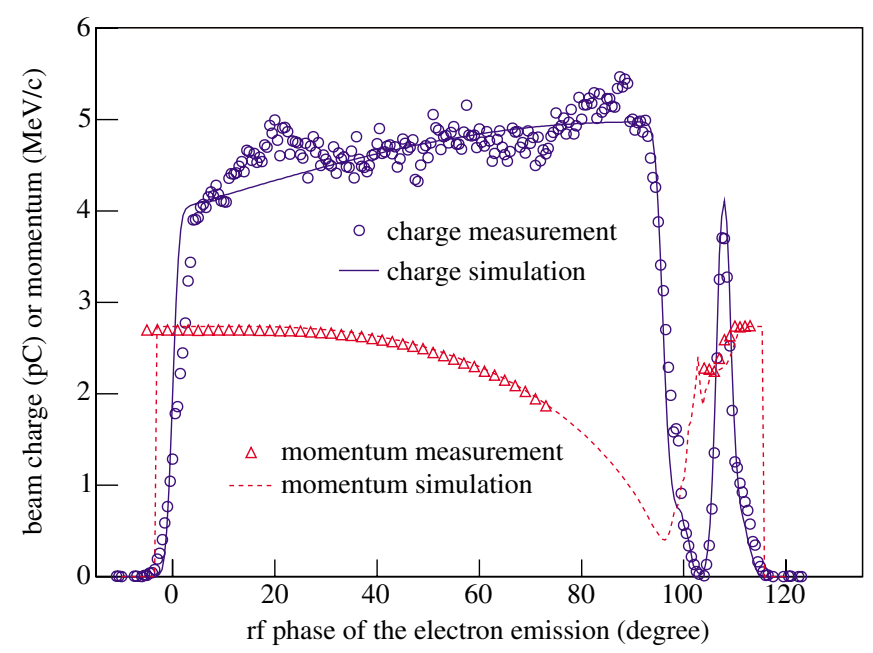

FIG. 10. (Color) Beam charge and momentum as a function of the emission phase. 
aluminum garnet (YAG) screen in the dispersive section have been used. A special control software [22] made it possible to combine images taken at various dipole settings in order to effectively increase the momentum acceptance of the spectrometer for the measurements.

Compared to the single electron dynamics discussed in the previous paragraph, additional effects have to be taken into account.
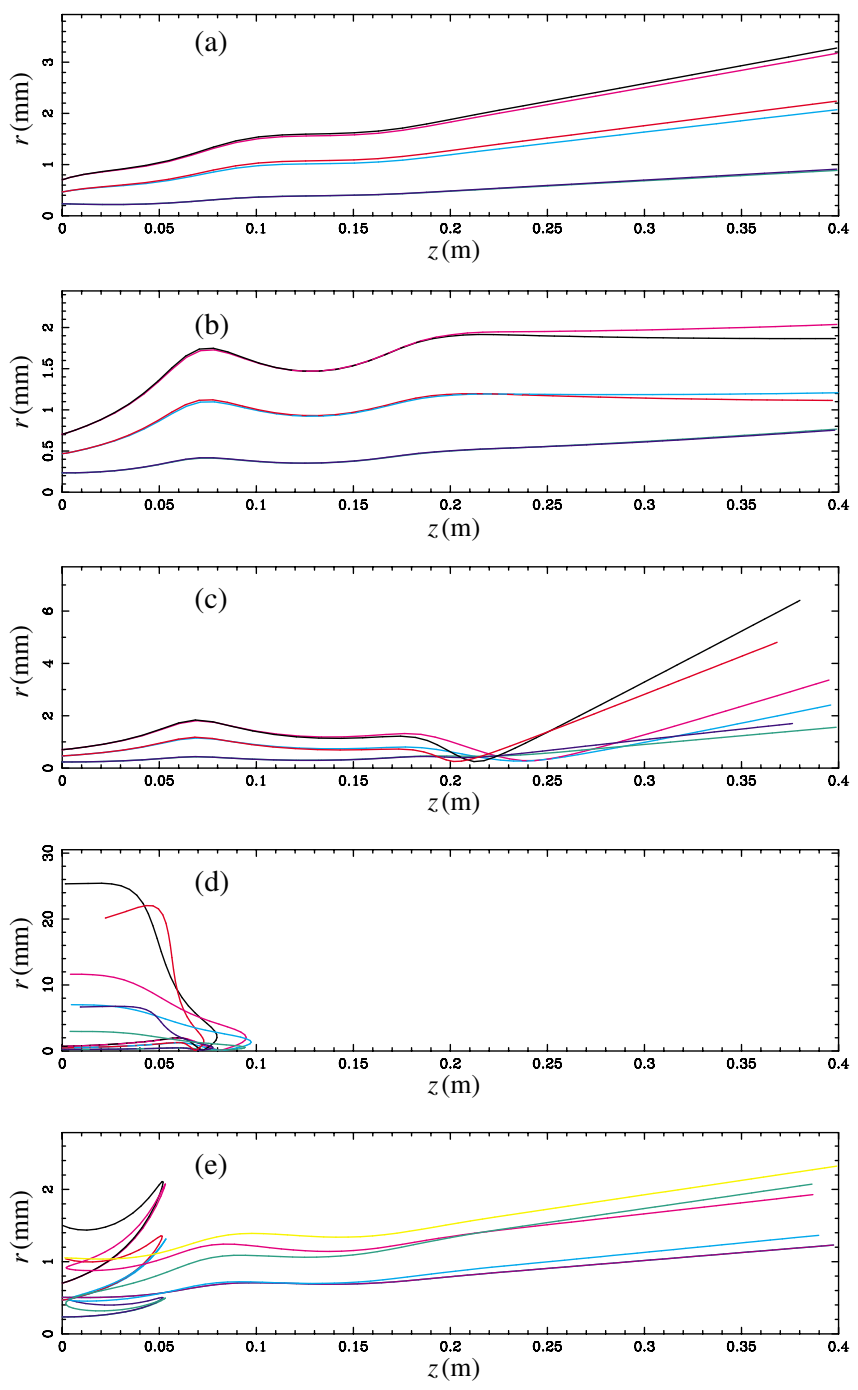

FIG. 11. (Color) Beam trajectories at characteristic emission phases. At $5^{\circ}$ (a) the electron beam can be fully extracted and smoothly accelerated because it is synchronized to the rf field in the full cell and hence gains its maximum momentum. At $90^{\circ}$ (b) the electron beam is decelerated and then accelerated again in the full cell. At this phase the transverse rf focusing is optimal to send the beam down the beam line. At $96^{\circ}$ (c) particles get lost due to deceleration and overfocusing. At $104^{\circ}$ (d) all emitted electrons return and disappear in the backplane of the cavity. At $108^{\circ}$ (e) a fraction of the electrons moves back to the cathode, to disappear or to produce secondaries, depending on the impact energy. The other fraction of the primaries barely survives and flows out of the cavity. (i) The temporal length of the laser pulse wipes out sharp structures in the dependence of parameters on the emission phase. Fine structures in the phase range between $100^{\circ}$ and $120^{\circ}$ cannot be resolved. Around $0^{\circ}$ the tail of the electron bunch which is generated by the laser pulse can already be extracted, while the head cannot escape from the cathode.

(ii) The quantum efficiency of the cathode shows a gradient dependence due to the Schottky effect [23], therefore the charge extraction increases somewhat within the phase range $0^{\circ}-90^{\circ}$.

(iii) For high phases above $90^{\circ}$ the transverse rf focusing tends to overfocus the electron beam and a fraction of the electrons gets lost due to the limited aperture of the beam pipe. Figure 11 displays the beam trajectories at characteristic emission phases.

For the simulations of Figs. 10 and 11 a flattop transverse laser profile with a rms radius of $0.47 \mathrm{~mm}$ and a Gaussian temporal laser profile with a rms size of $2.2 \mathrm{ps}$ was used in order to generate the photoemitted electron beam. The measured transverse laser size was $0.44 \mathrm{~mm} \mathrm{rms}$ for the horizontal direction and $0.51 \mathrm{~mm} \mathrm{rms}$ for the vertical direction. The transverse laser profile was measured with a CCD camera (JAI model CV-M10 RS) in an image plane which has the same distance and optics to the laser source as the photocathode.

The temporal laser size was measured to be $\sim 2.3 \mathrm{ps} \mathrm{rms}$ with a streak camera which has 2 ps resolution [24]. For the beam charge dependence on the rf phase, a Schottky effect was modeled with the formula [23] $Q \propto$ $\left(4.0+0.045 E_{\mathrm{rf}+\mathrm{sc}}[\mathrm{MV} / \mathrm{m}]\right)[\mathrm{pC}]$, where $E_{\mathrm{rf}+\mathrm{sc}}$ is the actual electric field ( $\mathrm{rf}+$ space charge) in the center of the cathode.

The charge bump between $100^{\circ}$ and $120^{\circ}$ is composed of both primary and secondary electrons, as shown in Fig. 12.

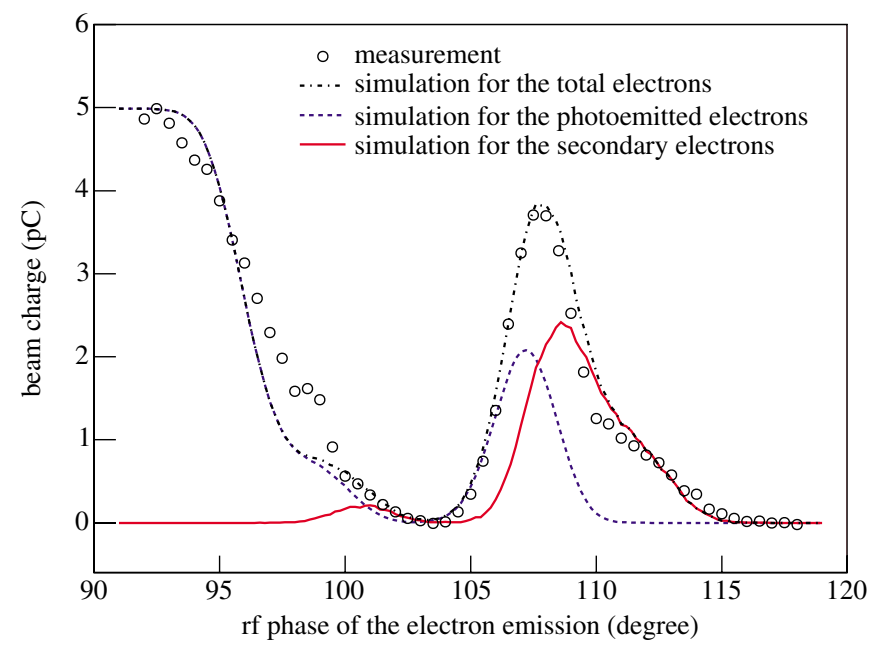

FIG. 12. (Color) Beam charge at the bump. In the simulation, the primary electrons and the secondary electrons are distinguished. 


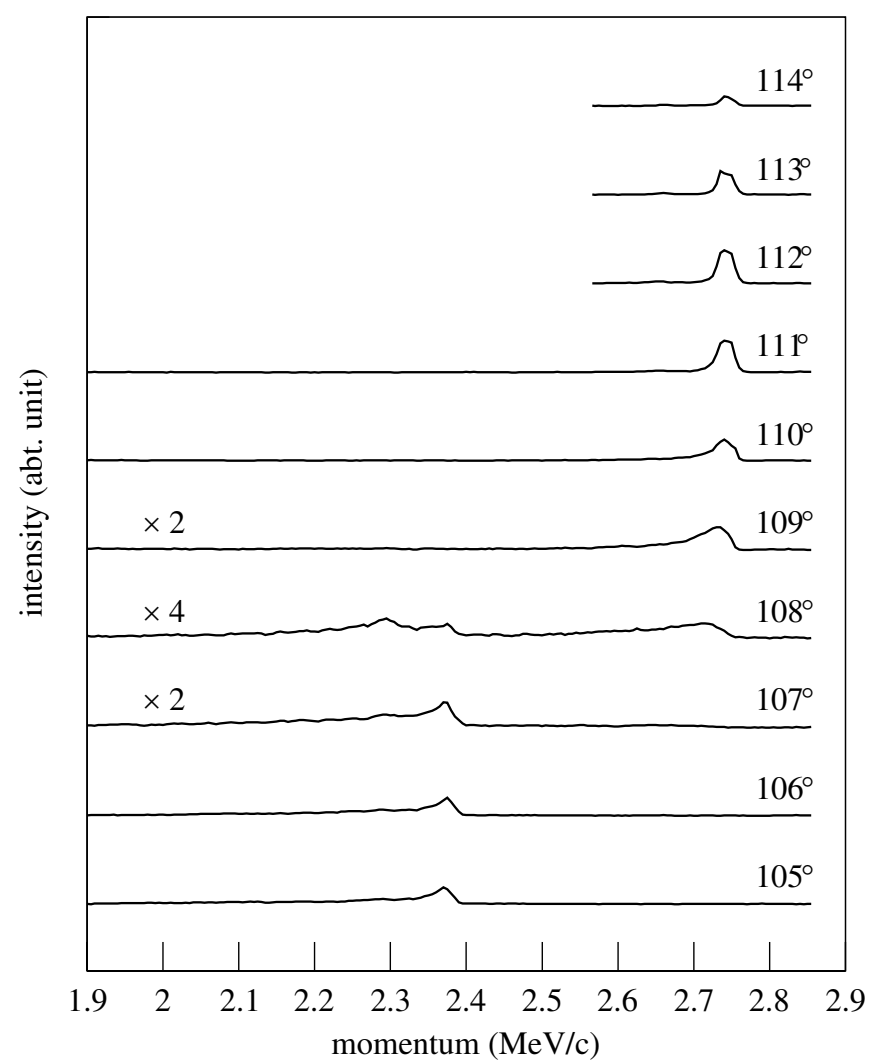

FIG. 13. Series of momentum measurements for the phase range $105^{\circ}-114^{\circ}$.

The momentum distributions of the bump as a function of the emission phase was investigated experimentally. A main solenoid field of $0.075 \mathrm{~T}$ (peak value) was applied in order to focus the electron beam onto the YAG screen in the dispersive section. The measured momentum distributions at a series of phases are plotted in Fig. 13. Two characteristic momenta were detected. For the lower phases $\left(105^{\circ}\right.$ and $106^{\circ}$ ) the beam momentum is $\sim 2.37 \mathrm{MeV} / c$. For the higher phases $\left(111^{\circ}-114^{\circ}\right) \sim 2.74 \mathrm{MeV} / c$ has been measured. Between $107^{\circ}$ and $109^{\circ}$, a continuous transition of the height of the two peaks from the lower momentum peak to the higher momentum peak has been observed.

Simulations were made to study this splitting of the momentum distribution (Fig. 14). The simulations show that the lower momentum peaks have their origin in electrons produced directly by the laser pulse and the higher momentum peaks originate from the secondary electrons. These secondary electrons have a higher momentum because the emission phases of the secondary electrons (i.e., impact phases of the primary electrons) correspond to the rf phases for maximum momentum gain (see Fig. 9). Broader peaks in the momentum measurements than in the simulations correspond to a rf power jitter of about $2 \%$ during the accumulation of the electron beam images on the YAG screen. For each measurement 50 electron bunch images were integrated on the YAG screen.

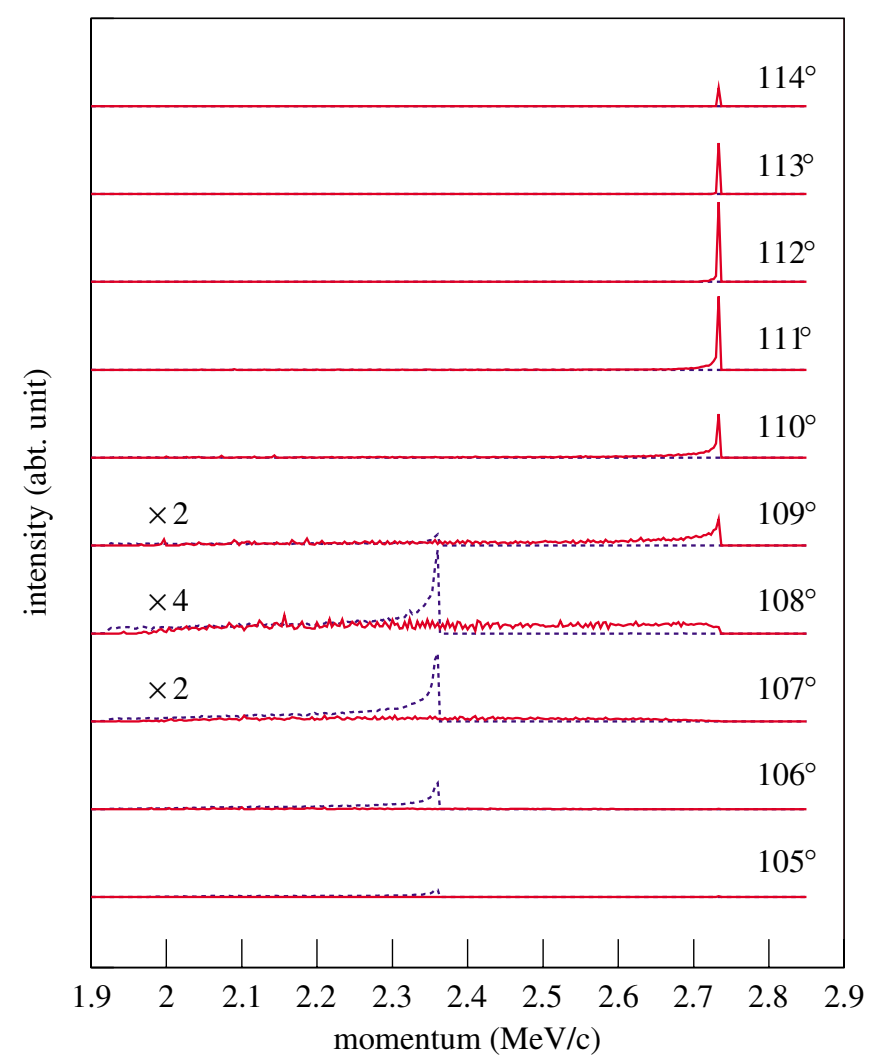

FIG. 14. (Color) Series of simulations of the momentum distribution in the phase range $105^{\circ}-114^{\circ}$. The electrons generated by the laser pulse (blue, dashed lines) and the secondary electrons (red, solid lines) are shown separately.

\section{DEPENDENCE ON CATHODE PARAMETERS}

In order to start to study the dependence of the secondary emission properties on cathode parameters, a second cathode, \#61 [20], has been used for additional charge and momentum measurements. Cathode \#61 has been produced following the same procedure and with the same geometry as cathode \#500 except for the $\mathrm{Cs}_{2} \mathrm{Te}$ film thickness, which is only $30 \mathrm{~nm}$ as compared to $60 \mathrm{~nm}$ for cathode \#500. The quantum efficiency of cathode \#61 was only $\sim 30 \%$ of the quantum efficiency of cathode \#500 during the measurements. To compensate the difference of the quantum efficiency of the two cathodes, the driving laser energy has been adjusted so that the bunch charge in the secondary-independent phase range, between $0^{\circ}$ and $90^{\circ}$, is the same for both cathodes.

The charge in the bump between $100^{\circ}$ and $120^{\circ}$ was found to be lower for cathode \#61. Figure 15 compares the measurements of both cathodes in the phase range of interest and shows a simulation matched for cathode \#61. For the simulation the maximum secondary emission yield was reduced from 7.0, as it was used in the simulations discussed above, to 4.5. Momentum measurements on cathode \#61 (see Fig. 16) show the expected behavior: The principle characteristics are identical to cathode 


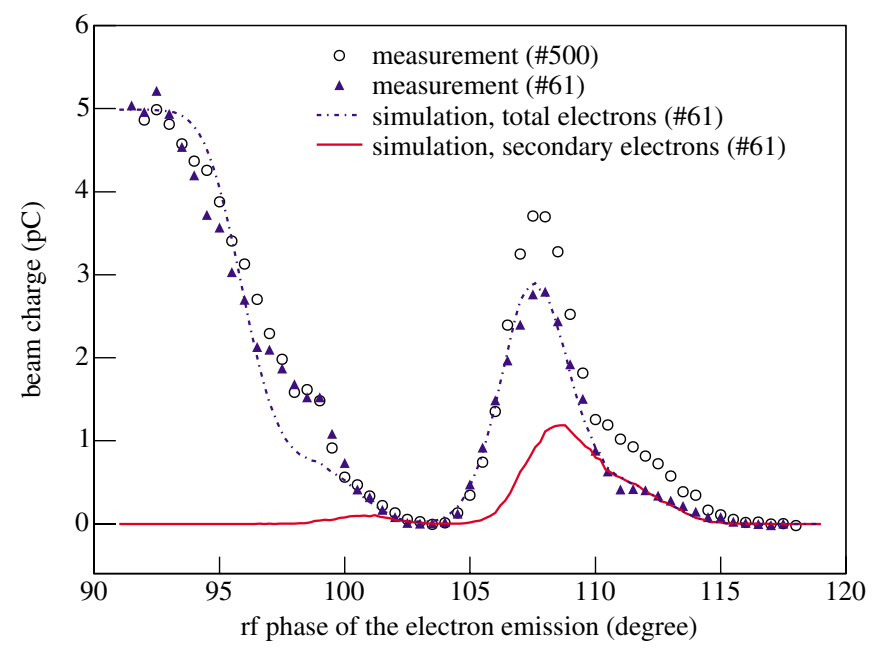

FIG. 15. (Color) Comparison of the bunch charges at the bump for cathode \#61 and cathode \#500.

$\# 500$, but the measured intensity at the higher momentum peak is lower for cathode \#61.

The cathode with the thicker $\mathrm{Cs}_{2} \mathrm{Te}$ coating is expected to have a higher secondary emission yield especially for primary electrons with impact energies higher than $1 \mathrm{keV}$ [25]. The lower quantum efficiency of cathode \#61 may be related to the cathode thickness, which is in the order of the photon absorption length as well as the electron escape depth, but may also be influenced by the surface status of

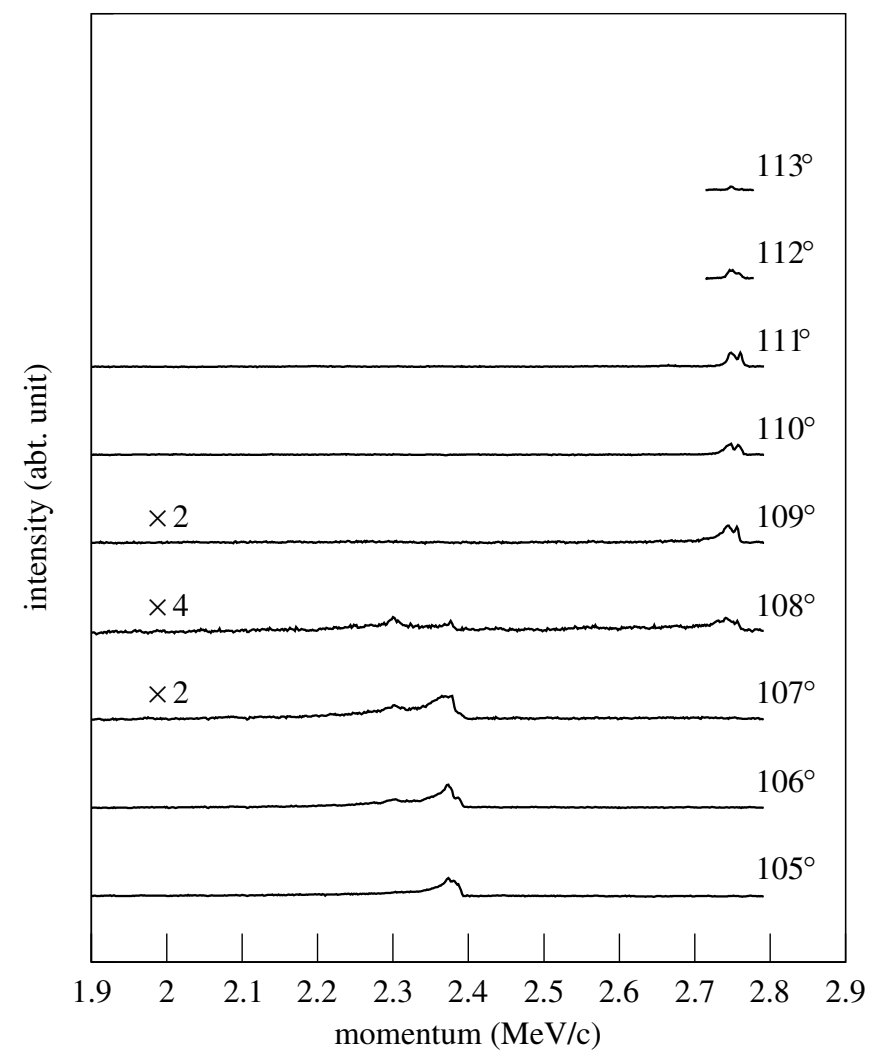

FIG. 16. Series of momentum measurements for cathode \#61. the cathode. $\mathrm{Cs}_{2} \mathrm{Te}$ has a low electron affinity of $\sim 0.2 \mathrm{eV}$, which allows the photoexcited electrons to easily overcome the potential barrier at the surface. Adsorbates on the cathode surface, which built up due to the vacuum conditions in the gun, can increase the electron affinity and hence reduce the quantum efficiency. As discussed in Sec. II, the electron affinity plays an important role in the secondary emission process as well.

\section{DISCUSSION AND CONCLUSION}

The measurements presented in this paper were obtained under conditions which allowed a clear observation of the secondary electrons. This allowed us to test the simplified model for the secondary emission which was implemented into the program ASTRA and to find appropriate parameters for the secondary emission yield of $\mathrm{Cs}_{2} \mathrm{Te}$ in a photocathode rf gun.

Secondary electrons are produced when the primaries start in a phase range which is also covered by dark current electrons, i.e., a fraction of the dark current electrons will hit back to the cathode and produce secondary electrons. From a numerical simulation with the parameters of $\mathrm{Cs}_{2} \mathrm{Te}$ used in this paper, the contribution of the secondaries to the dark current leaving the gun is estimated to be maximal $4 \%$ at normal operation conditions. The observations of Hartung et al. indicate, however, that the backbombardment characteristics depend also on the solenoid settings, which has been neglected in the present study. The results presented here are the basis for a further detailed investigation of the secondary emission effects in rf guns under various operation conditions which the authors intend to perform in the near future.

\section{ACKNOWLEDGMENTS}

The authors thank J. Bähr, I. Bohnet, U. Gensch, H.-J. Grabosch, V. Miltchev, A. Oppelt, B. Petrossyan, S. Riemann, J. Rönsch, J. Rossbach, P. Schmüser, S. Schreiber, L. Staykov, and F. Stephan (DESY), and M. von Hartrott, D. Lipka, and D. Richter (BESSY) for valuable discussions and experimental help. We are grateful to P. Michelato, L. Monaco, and D. Sertore (INFN Milan) for the cathode preparation. We are indebted to $\mathrm{K}$. Abrahamyan, G. Asova, G. Trowitzsch, and M. Winde for the data acquisition, W. Köhler, T. Thon, and R. Wenndorff for useful discussions about the rf system, and J. Binge, A. Donat, H. Lüdecke, M. Pohl, and F. Tonisch for technical support.

[1] V. Ayvazyan et al., Phys. Rev. Lett. 88, 104802 (2002).

[2] L. H. Yu et al., Phys. Rev. Lett. 91, 074801 (2003).

[3] K. J. Kim, Nucl. Instrum. Methods Phys. Res., Sect. A 275, 201 (1989). 
[4] B. E. Carlsten, Nucl. Instrum. Methods Phys. Res., Sect. A 285, 313 (1989).

[5] K. Flöttmann, ASTRA User's Manual, http://www.desy.de/ mpyflo/Astra_dokumentation

[6] L. Young and J. Billen, in Proceedings of the 2003 Particle Accelerator Conference, Portland, OR, 2003 (IEEE, Piscataway, NJ, 2003), p. 3521.

[7] W. Hartung, J.-P. Carneiro, D. Edwards, H. Edwards, D. Finley, M. Fitch, M. Kuchnir, D. Sertore, and P. Michelato, in Proceedings of the 2001 Particle Accelerator Conference, Chicago, IL, 2001 (IEEE, Piscataway, NJ, 2001), p. 2239.

[8] R. Brinkmann, Y. Derbenev, and K. Flöttmann, Phys. Rev. ST Accel. Beams 4, 053501 (2001).

[9] J.M. Hyatt and H.A. Smith, Phys. Rev. 32, 929 (1928).

[10] M. A. Furman and M. T.F. Pivi, Phys. Rev. ST Accel. Beams 5, 124404 (2002).

[11] A. Shih, J. Yater, C. Hor, and R. Abrams, Appl. Surf. Sci. 111, 251 (1997).

[12] R.C. Alig and S. Bloom, Phys. Rev. Lett. 35, 1522 (1975).

[13] A. H. Sommer, J. Appl. Phys. 42, 567 (1971), and references therein.

[14] J. Schou, Phys. Rev. B 22, 2141 (1980).
[15] T. Boutboul, A. Akkerman, A. Gibrekhterman, A. Breskin, and R. Chechik, J. Appl. Phys. 86, 5841 (1999).

[16] G. Diemer and J. L. H. Jonker, Philips Res. Rep. 5, 161 (1950).

[17] K. I. Grais and A. M. Bastawros, J. Appl. Phys. 53, 5239 (1982).

[18] S. A. Schwarz, J. Appl. Phys. 68, 2382 (1990).

[19] S. H. Kong, J. Kinross-Wright, D. C. Nguyen, and R. L. Sheffield, J. Appl. Phys. 77, 6031 (1995).

[20] Information on the photocathodes is available at http:// wwwlasa.mi.infn.it/ttfcathodes/

[21] F. Stephan et al., in Proceedings of the 26th International FEL Conference and 11th FEL Users Workshop, Trieste, Italy, 2004 (to be published); more information available at PITZ homepage: http://desyntwww.desy.de/pitz/

[22] D. Lipka, DESY PITZ Note 01-04, 2004.

[23] G. Suberlucq, in Proceedings of the 18th International FEL Conference, Rome, 1996 (Elsevier Science B.V., Amsterdam, 1997), p. II-131.

[24] The Hamamatsu streak camera module consists of a C5680 streak unit, a M5675 synchroscan sweep unit with a C4742-95 digital camera, and a M5679 dual time base extender unit.

[25] A. Akkerman, A. Gibrekhterman, A. Breskin, and R. Chechik, J. Appl. Phys. 72, 5429 (1992). 